International Journal of Medical Science and Clinical Research Studies

ISSN(print): 2767-8326, ISSN(online): 2767-8342

Volume 01 Issue 10 December 2021

Page No: $334-340$

DOI: https://doi.org/10.47191/ijmscrs/v1-i10-05, Impact Factor: 5.276

\title{
Role of Super Immune Activation Secondary to Infection in the Context of Cancer
}

\author{
Javier Contréras-Cárdenas ${ }^{1}$, José Maria Zepeda-Torres ${ }^{2}$, Felix Osuna-Gutiérrez ${ }^{3}$, Melany Jiménez- Brizuela ${ }^{4}$, \\ Walter Marroquín-Sandoval ${ }^{5}$, Mauricio Montelongo Quevedo ${ }^{6}$ \\ ${ }^{1}$ Faculty of Psychology of the National Autonomous University of Mexico \\ ${ }^{1,2,3,4,5,6}$ Faculty of Medicine of the Autonomous University of Guadalajara
}

\section{SUMMARY}

By 2008, it was estimated that there were about 12.7 million new cases of cancer worldwide, resulting in 7.6 million deaths. We are aware of the heterogeneity that exists and that it is impossible to link its development in any organ to a single pathophysiological mechanism. The greatest risk factor for developing cancer is aging, as age is directly proportional to accumulated aberrations and exposure to carcinogens. Most cancers occur in people who have no overt immunodeficiency. It is evident, then, that tumor cells must develop mechanisms to escape or evade the immune system in immunocompetent hosts. The main mechanisms of innate immunity against viruses are inhibition of infection by type I interferons and NK cell-mediated death of infected cells. The hypothesis is that perhaps the ability of epigenetic modification, which varies from virus to virus, is not exclusively reduced to the ability to activate genes that lead to cancer; but also randomly empower the organism to activatetumor suppressor genes.

KEYWORDS: Immune Activation, Cancer.

ARTICLE DETAILS
Published On:
14 December 2021
Available on:
https://ijmscr.org

\section{INTRODUCTION}

By 2008, it was estimated that there were about 12.7 million new cases of cancer worldwide, resulting in 7.6 million deaths (about 21,000 deaths per day). Taking into account the exponential increase in the size and age of the world's population, by 2030 it is projected that the number of cancer cases and cancer-related deaths worldwide will increase to 21.4 million and 13.2 million, respectively; in countries such as Mexico, lung, stomach and liver cancers are the most frequent in males, while breast, cervical and lung cancers are the most frequent in females.

Regarding the etiopathogenesis of cancer, we are aware of the heterogeneity that exists and that it is impossible to link its development in any organ to a single pathophysiological mechanism; age, infectious agents, smoking, alcoholism, diet, obesity, exposure to estrogens and carcinogens, are some of the agents that could cause a disruption in the delicate balance necessary to keep us free of cancer.

Thanks to our growing understanding of the molecular basis, we have further elucidated the importance of genetic and epigenetic alterations and their fundamental principles, as well as the features of mutated cells that give them an 334 Volume 01 Issue 10 December 2021 evolutionary advantage over their healthy counterparts, such as self-sufficiency, insensitivity to inhibitors, Warburg effect, apoptosis evasion, telomere maintenance, angiogenesis, metastatic capacity, and finally, the ability to evade recognition and regulation by the immune system. Returning to this last principle, tumors can be divided into "hot" tumors or "cold" tumors, depending on the type of immune response present and the type of inflammation caused by it. Hot" tumors are those in which the immune response is more active, with greater infiltration of adaptive immune response cells and pro-inflammatory cytokines, while "cold" tumors are those in which this response is absent or diminished, but each has certain microenvironmental conditions that may well help control and eliminate cancer, yet find ways to suppress the immune system [2]. Due to our understanding of the immune system, is that the importance of the theory that is about to be presented below is established, and is that the antiviral response seems to antagonize the effects of cancer itself for immune evasion, because they seem to share some metabolic pathways, is then where lies the importance of this issue, since from here, it is expected that new Corresponding Author: Walter Marroquí Sandoval 


\section{Role of Super Immune Activation Secondary to Infection in the Context of Cancer}

therapeutic targets are found, as well as strategies to combat the different types of cancer that afflict populations, to provide a better quality of life for patients who suffer from them.

\section{THEORETICAL FRAMEWORK}

Cancer is not a disease per se, rather it is the sum of disorders with complex and heterogeneous natural histories.

It is necessary to value cancer as a genetic disease in order to understand it, since an accumulation of detrimental variations in the human genome will lead to ontogenesis.

We all share the same genome; it is only the $0.5 \%$ variation in particular genomes that gives rise to individuality; most genetic mutations are benign, and tend not to be relevant. However, mutations to certain genes are critical.

It can be said that there are only two types of genes that have to do with our probability of developing cancer: oncogenes and tumor suppressor genes. Broadly speaking, the former promote cancer and the latter protect us from cancer.

Mutations that contribute to the development of a malignant phenotype are called driver mutations. Loss-offunction mutations in genes that maintain genomic integrity appear to be an early common denominator on the path to malignancy; once established, tumors evolve genetically during their growth and progression under the pressure of Darwinian selection; in addition to DNA mutations, epigenetic aberrations also contribute to the malignant properties of cancer cells.

The greatest risk factor for developing cancer is aging, as age is directly proportional to accumulated aberrations and exposure to carcinogens.

There are two ways in which mutations have an impact: somatic mutation and germlinemutation. Somatic mutation is achieved through DNA damage by environmental factors (repeated exposure to UV rays, alcohol, smoking, among others) or by errors during the replication of genetic information; the somatic cell to reproduce transmit damage to their offspring, but can never be inherited to new genealogical lines. On the other hand, there are germline mutations, which are responsible for certain types of cancer in several members of the same family. An example is the BRCA1/2 genes and breast cancer in entire families, but it should be noted that not being a woman, and having this gene, is a sentence of breast cancer, because it requires more than one mutation.

It should be noted that humans are diploid, we have a maternal and a paternal copy of the genes, this means that the mutation of an allele does not condition the correct functioning of the specific gene; both copies have to be affected to induce cancer, or the DNA or its expression has to be directly affected by an epigenetic mechanism.

Most cancers require multiple mutations in a variety of different genes in order to takeplace. For a cell to become cancerous it must have a minimum of 6 to 7 mutations in multiple genes; it usually requires inactivation of tumor suppressor genes and simultaneous activation of oncogenes -double hit effect- to initiate cancer. Onco-cells present genomic instability, this means that the cells begin to survive and reproduce despite having high mutation rates compared to regular cells, and subsequently this will lead to an exponential growth of the range of genetic mutations. Minimal etiopathogenic factors necessary for the development of cancer:

1. Self-sufficiency in growth

2. Insensitivity to anti-growth signals

3. Avoidance of apoptosis

4. Unlimited replication potential

5. Sustained angiogenesis

6. Tissue invasion and metastasis

In the pathophysiology of cancer we find 8 fundamental principles, which are considered pathognomonic. These are listed as follows:

1. Self-sustaining in mitogens and growth factors.

2. Insensitivity to growth inhibitory signals.

3. Altered cellular metabolism (Warburg effect)

4. Avoidance of apoptosis.

5. Unlimited mitotic potential.

6. Angiogenesis.

7. Ability to invade and metastasize.

8. Evasion of the host immune response.

Under the assumption that cancers occur even in immunocompetent individuals, it is understood that immune surveillance is imperfect; however, the fact that some tumorsescape such surveillance does not exclude the possibility that many others were suppressed by recognition of tumor antigens resulting from altered cellular metabolism or genome, which will be described below.

The main classes of tumor antigens are as follows:

1. Products of mutated genes. Thanks to genetic instability, cancers have a high burden of mutations throughout their genomes, some of these transient mutations can fall into the coding sequences of genes and give rise to aberrantproteins that serve as tumor antigens, and like any cytoplasmic protein, can enter the MHC class I antigen processing pathway and be recognized by $\mathrm{CD} 8+\mathrm{T}$ cells. In addition, these proteins can enter the class II antigen processing pathway on antigen-presenting cells that have phagocytosed dead tumor cells and thus can also be recognized by $\mathrm{CD} 4+\mathrm{T}$ cells.

2. Protein overexpression or aberrant expression. Tumor antigens can also be normal cellular proteins that are overexpressed. Another group of tumor antigens are

Those that under normal circumstances are sequestered in Immune privileged sites, therefore, their appearance outside of these confinements is considered abnormal.

3. Tumor antigens produced by oncogenic viruses. The most potent of these antigens are proteins derived 


\section{Role of Super Immune Activation Secondary to Infection in the Context of Cancer}

from latent DNA viruses;

4. Oncofetal antigens.Oncofetal antigens are proteins that are expressed at high levels in cancer cells and in normal developing fetal tissues.

5. Surface glycolipids and glycoproteins. Most human and experimental tumors express higher than normal levels and abnormal forms of surface glycoproteinsand glycolipids [6].

6. Cell type-specific differentiation antigens. They are so named because they arespecific for particular lineages or stages of differentiation of various cell types. Such differentiation antigens are typically normal selfantigens and therefore donot induce immune responses in tumor-bearing hosts.

\section{GENE REGULATION AND PATHOGENS}

Before continuing it is also important to return to the idea of genetics in cancer and the important role that viruses can play in this. Gene regulation is the process by which the cell determines a set of genes and how they function, that is, it turns genes off andon according to organic need; it is a device that ensures that the right genes are expressed to help the organism respond to the environment.

Genes can be classified with respect to their role in protein synthesis into two major groups: structural and regulatory; the former contain coding information; while regulators, which lack coding information, but order and regulate, determine transcription timing, or have specific roles during mitosis and meiosis, or denote wherethe active sites will be.

When viruses cause an infection, they spread their DNA, affecting the genetic makeupof healthy cells and potentially causing them to develop into cancer. HPV infections, for example, cause the virus's DNA to combine with the host's DNA, disrupting the normal functioning of cells.

Human oncogenic viruses have diverse genomes, cell tropisms, cancer pathologies and disease prevalence. However, they share many characteristics that can cause cancer in humans. They are transmitted between humans and can establish chronic infections that last for years without obvious symptoms. Over these prolonged periods, oncogenic viruses co-opt cellular processes for replication and undermine immune recognition. They derail conserved signaling pathways that control cell cycle progression and apoptosis to support their spread.

\section{IMMUNE SURVEILLANCE AND ESCAPE}

Most cancers occur in people who have no overt immunodeficiency. It is evident, then, that tumor cells must develop mechanisms to escape or evade the immune system in immunocompetent hosts. The mechanisms are discussed below: Selection of antigen-negative variants. During tumor progression, highly immunogenic clones are eliminated.

1. Reduced or absent expression of MHC molecules. Tumor cells may not express normal levels of HLA class I molecules, thus escaping attack by cytotoxic $\mathrm{T}$ cells. Paradoxically, however, this may activate NK cells if the tumor cells express ligands for NK cell activation receptors since the absence of HLA type I receptors activates these cells [4].

2. Activation of immunoregulatory pathways. A concept that is now gaining currency is that tumor cells actively inhibit tumor immunity by activating normal immune regulatory pathways that serve as "checkpoints" in immune responses. Through a variety of mechanisms, tumor cells can negatively regulate the expression of co-stimulatory factors on antigen-presenting cells, such as dendritic cells; as a result, antigen-presenting cells fail to bind to the stimulatory receptor CD28 and instead activate the inhibitory receptor CTLA-4 on effector $\mathrm{T}$ cells. This not only prevents sensitization, but can also induce energy. Tumor cells can also positively regulate the expression of PD-L1 and PDL2, cell surface proteins that activate the programmed death receptor-1 (PD-1) on effector T cells. PD-1, like CTLA-4, can inhibit $\mathrm{T}$ cell activation [2].

3. Secretion of immunosuppressive factors. Tumors can secrete various products that inhibit the host immune response. Many tumors secrete TGF- $\beta$ in large amounts and is a potent immunosuppressant. Other tumors secrete gale tins, sugar-rich lectin-like factors that bias $\mathrm{T}$ cell responses to favor immunosuppression [6]. Many other soluble factors produced by tumors are also suspected to inhibit the host immune response, including interleukin-10, prostaglandin E2, certain tryptophan-derived metabolites, and VEGF, which may inhibit T-cell diapedesis from the vasculature to the tumor bed. And finally, to prevent infiltration of cytotoxic $\mathrm{T}$ cells they also generate a microenvironmentwhere the availability of oxygen is more reduced and the density of the extracellular matrix is much higher, preventing immunosurveillance by stoppingimmune cells before they come in contact with tumor cells [2].

4. Induction of regulatory $\mathrm{T}$ cells (Tregs). Some studies suggest that tumors produce factors that promote the development of immunosuppressive regulatory $\mathrm{T}$ cells, which may also contribute to "immunoevasion". Thus, there appears to be no shortage of mechanisms by which tumor cells can circumvent the host immune system. Nevertheless, the aforementioned response of tumors to immunomodulatory agents, such as antibodies that block CTLA-4 and PD-1, has generated great excitement about the potential of modern cancer immunotherapy.

\section{ANTITUMOR EFFECTOR MECHANISMS}

Cell-mediated immunity is the dominant antitumor mechanism in vivo.

1. CD8+ lymphocytes. These have a clear protective role against virus-associated malignancies, and several studies have shown that the number of tumorinfiltrating $\mathrm{CD} 8+\mathrm{T}$ cells and the presence of a 
Role of Super Immune Activation Secondary to Infection in the Context of Cancer

"genetic signature" associated with CD8+ CTLs is directly associated with a better prognosis in a variety of cancers, not only those caused by oncogenic viruses [3].

2. Natural killer cells. NK cells are lymphocytes capable of destroying tumor cells without prior sensitization and thus provide the first line of defense against tumor cells. Once activated with IL-2 and IL-15, NK cells can lyse a wide variety of human tumors, even those that appear to be non-immunogenic to $\mathrm{T}$ cells.

3. Macrophages. Activated macrophages exhibit cytotoxicity against tumor cells in vitro. T cells, NK cells, and macrophages may collaborate in antitumor reactivity, because interferon- $\gamma$, a cytokine secreted by $\mathrm{T}$ cells and NK cells, is a potent activator of macrophages. Activated macrophages can kill tumors by mechanisms similar to those used to kill microbes.

\section{IMMUNITY TO VIRUSES}

Viruses are obligate intracellular microorganisms that use components of the host's synthetic machinery of nucleic acids and proteins to replicate and propagate. After entering cells, they can cause tissue injury and disease by any of several mechanisms. Viral replication interferes with the synthesis and function of normal cellular proteins and leads to injury and ultimately death of the infected cell. This result is a type of cytopathic effect of viruses, and the infection is said to be lytic because the infected cell is lysed.

Innate and adaptive immune responses to viruses aim to block infection and eliminate infected cells. Type I interferons prevent infection as part of innate immunity and neutralizing antibodies contribute to adaptive immunity. Once infection is established,NK cells eliminate infected cells in the innate response and CTLs in the adaptive response.

The main mechanisms of innate immunity against viruses are inhibition of infection by type I interferons and NK cell-mediated killing of infected cells [4]. The main effector of the innate response to counteract viral infection is the production of type 1 IFN, mostly by infected cells, following a signaling cascade caused by recognition of viral RNA or DNA within cells and by surface Toll-like receptors. Dendritic cells of the plasmacytoid type are also major producers of this interferon. The various biochemical pathways that trigger interferon production include recognition of viral RNA and DNA by endosomal TLRs and activation of cytoplasmic RIG-like receptors and the STING pathway by viral RNA and DNA, respectively. These pathways converge in the activation of protein kinases, which in turn activate IRF transcription factors that stimulate interferon gene transcription. Type I interferons function to inhibit viral replication in both infected and uninfected cells.

Type I interferons, which signal through the type I interferon receptor, activate the transcription of several genes that give cells a resistance to viral infection called the antiviral state. Type I interferon-induced genes include the double-stranded RNA- activated serine/threonine protein kinase (PKR), which blocks viral transcription and translation events, and oligoadenylate synthetase 2', 5' and L-RNAase, which promote viral RNA degradation. The antiviral action of type I interferon is primarily a paracrine action in which a virus-infected cell secretes interferon to act on and protect neighboring cells that are not yet infected. The effects of type 1 interferons are not specific to viral gene expression, and part of the ability of these cytokines to block the

Spread of infection is due to their toxicity to host cells in the vicinity of infected cells. Interferon secreted by an infected cell can also act in an autocrine manner to inhibit viral replication in that cell.

Type I interferons cause lymphocyte sequestration in lymph nodes, which maximizes the opportunity to encounter microbial antigens. The mechanism of this effect of typeI interferons is the induction of a molecule on lymphocytes, called CD69, which forms a complex with the sphingosine 1-phosphate (S1P) receptor S1PR1 and reduces surface expression.

Type I interferons increase the cytotoxicity of NK cells and $\mathrm{CD} 8 \mathrm{CTLs}^{+}$and promote differentiation of virgin $\mathrm{T}$ cells to the $\mathrm{T} H 1$ helper $\mathrm{T}$ cell subset.

Type I interferons positively regulate the expression of MHC class I molecules and thus increase the likelihood that virus-infected cells will be recognized and killed by CD8 CTLs. ${ }^{+}$Virus-specific CTLCD8s ${ }^{+}$recognize peptides derived from viral proteins bound to MHC class I molecules on the surface of infected cells. Therefore, by increasing the amount of class I MHC synthesized by a virus-infected cell, type I interferons will increase the number of viral peptide class I MHC complexes on the cell surface that CTLs can see and respond to. The end result is the destruction of cells that support viral replication, which is necessary to eradicate viral infections.

NK cells kill other cells infected with a variety of viruses and are an important mechanism of immunity against viruses early in the course of infection, before adaptive immune responses develop. MHC class I expression is often disrupted in virus-infected cells as an escape mechanism from CTLs. This allows NK cells to eliminate infected cells because the absence of class I releases NK cells from a normal state of inhibition.

\section{ADAPTIVE IMMUNITY TO VIRUSES}

Adaptive immunity against viral infections is mediated by antibodies, which block virus binding and entry into host cells, and by CTLs, which eliminate infection by destroyinginfected cells. The most effective antibodies are high-affinity antibodies produced in T-dependent 


\section{Role of Super Immune Activation Secondary to Infection in the Context of Cancer}

germinal center reactions. Antibodies are effective against viruses only during the extracellular stage of the life of these microbes. Viruses may be extracellular early in the course of infection, before they infect host cells, or when theyare released from infected cells by virus budding or if infected cells die. Antiviral antibodies bind to viral envelope or capsid antigens and function primarily as neutralizing antibodies to prevent virus binding and entry into host cells. Antibodies therefore prevent both initial infection and cell-to-cell spread.

Clearance of viruses residing within cells is mediated by CTLs, which kill infected cells. Most virus-specific CTLs are TCD8 cells ${ }^{+}$that recognize cytosolic viral peptides, usually endogenously synthesized, presented by MHC class I molecules [5]. If the infected cell is a tissue cell and not a professional antigen presenting cell (APC), suchas a dendritic cell, the infected cell can be phagocytosed by the dendritic cell, which processes viral antigens and presents them to naïve $\mathrm{CD} 8^{+} \mathrm{T}$ cells. Complete differentiation of CD8 $\mathrm{CTLs}^{+}$often requires cytokines produced by CD4 helper cells

${ }^{+}$or co-stimulators expressed on infected cells CD8 T cells ${ }^{+}$undergo massive

Proliferation during viral infection and most proliferating cells are specific for some

Viral peptides. Some of the activated T cells differentiate into effector CTLs, which can kill any infected nucleated cell. The antiviral effects of CTLs are primarily due to the killing of infected cells, but other mechanisms include the activation of nucleases within infected cells that degrade viral genomes and the secretion of cytokines such as IFN$\gamma$. Which activates phagocytes and may have some antiviral activity.

The importance of CTLs in defense against viral infections is demonstrated by the increased susceptibility to such infections observed in patients and animals with $\mathrm{T}$ lymphocyte deficiency and by the experimental observation that mice can protect themselves against some viral infections by adoptive transfer of specific classes of restricted CTLs. In addition, many viruses can alter their surface antigens, such as envelope glycoproteins, and thus escape antibody attack. However, infected cells canproduce some viral proteins that are invariant, so that CTL-mediated defense remainseffective against such viruses.

In latent infections, viral DNA persists in host cells, but the virus does not replicate or destroy infected cells. Latency is often a state of equilibrium between infection and immune response. CTLs are generated in response to the virus that can control the infection but not eradicate it. As a result, the virus persists in infected cells, sometimesfor the entire life of the individual. Any deficiency in the host immune response can result in reactivation of latent infection, with expression of viral genes that are responsible for cytopathic effects and viral shedding. These cytopathic effects may include lysis of infected cells or uncontrolled proliferation of cells. These latent infections are common in DNA viruses such as Epstein-Barr virus associated with B- lymphocyte lymphomas and nasopharyngeal carcinoma, human papillomavirus associated with cervical and oropharyngeal carcinoma, and herpesvirus associated with Kaposi's sarcoma to name a few.

\section{DISCUSSION}

Regarding the activity of the immune system in response to invasion and infection by viruses, we begin by mentioning that the cells of the innate immune system involved in the response against these microorganisms are NK lymphocytes, while the cells involved in the adaptive system are mainly CD8 or cytolytic T lymphocytes, both typesof cells involved in the surveillance and destruction of cancerous tumors. Additionally, one of the most important roles for the optimization of this cellular response is played by type I interferons such as interferon-y. Therefore, the different mechanisms by which the immune system is activated to attack the viral infection can in theory also stimulate the anti-tumor immune response, as these elements independently intervene to eliminate what they do not recognize as their own.

One of the strategies of tumor evasion of Immunosurveillance is precisely the reduction of the immunogenicity of neoplastic cells by affecting the mechanisms of antigen processing and presentation [5], as mentioned above. Both tumors and virus- infected cells manipulate the expression of human leukocyte antigens in an attempt to evade the adaptive immune response. The former do so because they express abnormal proteins or molecules that attract attention, viruses produce viral proteins that are totally foreign to our body and therefore hide this in an attempt to survive and multiply in greater numbers before they are discovered. This is a common fa between viruses and cancerous tumors that leads to the activation of NK lymphocytes. At this point the activity of the NK cells is seen as how it can help in one case or another but not really how the activation of these cells by the viral infection could help these cells become better at attacking and controlling the growing population of cells in a cancer tumor. It should be added that $\mathrm{T}$ lymphocytes, mentioned as the main participants of the adaptive cellular response in these cases, are "educated" in the thymus and subjected to a positive selection, against those lymphocytes with a null reactivity against class 1 self-molecules, which are eliminated, and negative for those lymphocytes that show a high reactivity. The result is a population of effectors with an intermediate affinity for the MHC-proper peptide complex. And the number of different subtypes of effector $\mathrm{T}$ lymphocytes also plays an important role because with their different functions and locations in the body they protect against the spread and proliferation of cancer, it has been found that patients have a better

Corresponding Author: Walter Marroquí Sandoval 


\section{Role of Super Immune Activation Secondary to Infection in the Context of Cancer}

prognosis when there are more lymphocytes of different subtypes in the affected and surrounding tissues than when there are none [3]. The recognition by cytotoxic lymphocytes of antigenic peptides that can begenerated in any cell of the organism is important for the recognition of intracellular pathogens, which are destroyed preventing their dissemination, or those cells that undergo tumor transformation and express mutated self-antigens or selfantigens not synthesized from very early stages of development [3]. This is something that helps us to further cement what was mentioned about the "shared" immune response against viruses and tumors, however it does not help us to answer in a concrete way how the adaptive response towards viruses could help to attack a different pathologicalentity when there is great specificity.

Taking into account the above information, the problem of specific activity of the cells involved is solved by the intermediary cells and cytokines that are involved in the activation of these cells. We can infer to some extent with the information gathered is that the activation of mature dendritic cells for both tumor and viral antigen presentation plays an important role in the immune response that is not directly involved with the destruction of the affected cells (the infected cells and tumor mutated cells) [5]. These cells, while they may be evaded by tumor cells normally, upon encountering virus-infected cells will begin to perform this described function by "waking up" macrophages, NK cells and lymphocytes. Dendritic cells interact with NK cells to promote the production of cytokines such as IL-12, IL-15, TNF and interferon-y [4]. Interferon-y among other things promotes the production and expression of MHC-I receptors on all cells so this cytokine released by viral infection may well help in the identification of tumor cells by being found in large quantities in the bloodstreamand thus reaching them easily. These dendritic cells could therefore find exposed cancer antigens and present them to TCD4 and TCD8 lymphocytes, having also IL-2 and IL-15 present promoting the proliferation and survival of antitumor $\mathrm{T}$ cells [3], overlapping the regulatory activities imposed by the tumor thanks to the hyperactivation caused by the viral infection and leading in turn to the recognition and destruction of cancer cells. However, it is important to take into account that it is not common to hear that a person with an infection improves in their oncologic disease, although this could be a way in which the immune system could be reactivated in its Antitumor activity we would have to perform more specific studies and look for more cases where this could be explained.

Also as mentioned above, human oncogenic viruses have genomes, cell tropisms, cancer pathologies and prevalence of other diseases and share many features that can cause cancer in humans due to major alterations at the level of the nucleus and the nucleic acids found therein. Although tumorigenesis is a unifying pathological feature for oncogenic viruses, it is neither evolutionarily advantageous for the virus nor necessary for virus propagation. We can therefore speak of epigenetics in which the virus interacts with the human genome in the cells it infects and from this idea we candevelop a hypothesis.

The concept of epigenetics has taught us that there are activations and deactivationsof genes throughout our lives, without necessarily having a structural change of the underlying DNA of the organism. Epigenetic sequences are usually stable throughout our days, however, environmental factors can affect them; these epigenetic modifications serve as an explanation of the ability of the environment to transform human characters through genotype-environment contact; this also happens with cancer.

There are several epigenetic processes described such as DNA methylation, histone chemical modification, chromatin remodeling and non-coding RNA synthesis.

Some viruses are able to induce neoplastic transformation through the integration of proviral DNA and the activation of host-specific genes: cellular genes are brought under the control of long repeated sequences of the virus or are fused to viral sequences, with the consequent production of novel proteins. RNA viruses or retroviruses, similar in structure to the human immunodeficiency virus or HIV, have a reverse transcriptase enzyme, responsible for the synthesis of DNA from RNA.

The provirus can affect the loci in the region of the host chromosome where it integrates. If a proto-oncogene is present in that region, provirus integration may alter its structure and/or expression. Some proto-oncogenes are affected by somatic mutations in tumors. Although viruses have been a tool to identify numerous genes involved in tumor genesis, only a small fraction of viruses are associated with human cancers and in most cases, they increase the risk of developing the tumor, rather thanbeing exclusive determinants of the disease.

It has been evidenced that cancer cells present global changes within the epigenome, involving complete signaling, growth and apoptosis pathways. Cancer types are associated with different epigenetic modifications that may play a relevant role in carcinogenesis: origin of carcinogenic cells and tumor progression. The influence of viruses in cancer has been extensively reported in the literature, even when they are considered as noncommunicable diseases.

An indication of the advantage of viral infections on the pathogenesis of cancer are the favorable results of oncolytic therapy, which today opens new avenues in immunotherapy against this disease.

The application of the first oncolytic T-EV virus for the treatment of unresectable metastatic melanoma in October 


\section{Role of Super Immune Activation Secondary to Infection in the Context of Cancer}

2015 is an extremely important event in terms of updating this type of therapeutic mechanism. (7)

Oncolytic therapy has two main effects, one is direct oncolysis, and the second is the invasion of oncolytic viruses into tumor cells, using their energy and raw material, blocking their growth and differentiation; finally leading to cell lysis, causing the releaseof products that will ultimately cause viral infection throughout the tumor mass. Viral replication leads to the death of immunogenic cancer cells by necrosis and necroptosis. (8)

This releases molecules that stimulate the maturation and activation of antigen- presenting cells, causing the release of tumor antigen-specific $\mathrm{T}$ cells; consequently infected tumor cells will be attacked by the adaptive and innate immune systems; it can also induce antivascular effects and alter the extracellular matrix enhancing viral dissemination.

Although oncolytic therapy shows great advances at present, it should be considered as a double-edged sword, since the host immune response can hinder viral replication and propagation, and thus add to the neoplastic pathology an active viral infection; the field in which more scientific bases are needed in terms of this type of therapy is the route of administration, it is currently considered that direct intertumoral injection should be the main route, but the ideal is to investigate the most effective to reduce side effects and maximize its benefits. (8)

\section{CONCLUSIONS}

The hypothesis is that perhaps the capacity of epigenetic modification, which varies from virus to virus, is not exclusively reduced to the capacity to activate genes that lead to cancer; but also randomly empower the organism to activate genes of the tumour suppressor type. When the cancer is already active and proliferating, it may be that the intrusion of a virus can aggravate the natural course of the pathology, or even improve the conditions of the immune system in its combat; a process which we will call immune super-activation secondary to viral infection, and which is capable of breaking the activated mechanism of the double whammy.

The authors of the present text consider this an interesting theory, however, we are aware that to date there is not enough evidence to establish a causal relationship that irrefutably supports this hypothesis; Even so, it is relevant to maintain a creative line of thought when dealing with pathologies as heterogeneous as those collected in the context of cancer, because its integral treatment is difficult, and although in recent times it is more hopeful in some cases, we are still far from being able to manage it successfully in most patients, and the fact of proposing these theories is the anteroom to develop new therapeutics and to be able to give an integral management to those who suffer from these diseases.

\section{BIBLIOGRAPHIC REFERENCES}

I. Kumar V, Abbas AK, Aster JC, Biernat W, Olszewski W, Perkins JA, et al. Robbins pathology. Wrocław: Edra Urban \&amp; Partner; 2019.

II. Armitage, J. D., Newnes, H. V., McDonnell, A., Bosco, A., \&amp; Waithman, J.(2021). Fine-tuning the tumour microenvironment: Current perspectives on the mechanisms of tumour immunosuppression. Cells, 10(1), 56. doi:10.3390/cells10010056.

III. Han J, Khatwani N, Searles TG, Turk MJ, Angeles $\mathrm{CV}$. Memory cd8+ $\mathrm{t}$ cell responses to cancer. Seminars in Immunology. 2020;49:101435.

IV. Taborda NA, Hernández JC, Montoya CJ, Rugeles MT. Natural killer cells and their role in the immune response during human immunodeficiency virus type- 1 infection. Immunology. 2014; 33(1):11-20.

V. de León J, Pareja A. Cancer immunology I: molecular and cellular basis of the antitumor immune response. Horizonte Médico (Lima). 2018;18(3):80-9.

VI. Espinoza-Sánchez NA, Götte M. Role of cell surface proteoglycans in cancer immunotherapy. Seminars in Cancer Biology. 2020;62:48-67.

VII. Sun, W., Shi, Q., Zhang, H., Yang, K., Ke, Y., Wang, Y., \& Qiao, L. (2019). Advances in the techniques and methodologies of cancer gene therapy. Discovery medicine, 27(146), 45-55.

VIII. Mohanty, R., Chowdhury, C. R., Arega, S., Sen, P., Ganguly, P., \& Ganguly, N. (2019). CAR T cell therapy: a new era for cancer treatment. Oncology reports, 42(6), 2183-2195. 